[5] J. K. Hale and S. M. V. Lunel, Introduction to Functional Differential Equations. New York: Springer-Verlag, 1993.

[6] L. Huang and F. Deng, "Razumikhin-type theorems on stability of neutral stochastic functional differential equations," IEEE Trans. Automat. Control, vol. 53, no. 7, pp. 1718-1723, Aug. 2008.

[7] L. Huang, X. Mao, and F. Deng, "Stability of hybrid stochastic retarded systems," IEEE Trans. Circuits Syst. I: Regular Papers, vol. 55, no. 11, pp. 3413-3420, Dec. 2008.

[8] Y. Ji and H. J. Chizeck, "Controllability, stabilizability and continuoustime Markovian jump linear quadratic control," IEEE Trans. Automat. Control, vol. 35, no. 7, pp. 777-788, Jul. 1990.

[9] H. K. Khalil, Nonlinear Systems, 3rd ed. Englewood Cliffs, NJ: Prentice Hall, 2002.

[10] S.-J. Liu, S. S. Ge, and J.-F. Zhang, "Adaptive output-feedback control for a class of uncertain stochastic non-linear systems with time delays," Int. J. Control, vol. 81, pp. 1210-1220, 2008.

[11] S.-J. Liu, Z.-P. Jiang, and J.-F. Zhang, "Global output-feedback stabilization for a class of stochastic non-minimum-phase nonlinear systems," Automatica, vol. 44, pp. 1944-1957, 2008.

[12] X. Mao, "Razumikhin-type theorems on exponential stability of stochastic functional differential equations," Stochastic Processes Appl., vol. 65 , pp. 233-250, 1996

[13] X. Mao, A. Matasov, and A. B. Piunovskiy, "Stochastic differential delay equations with Markovian switching," in Proc. Bernoulli Conf., 2000, vol. 6, pp. 73-90.

[14] X. Mao, "Exponential stability of stochastic delay interval systems with Markovian switching," IEEE Trans. Automat. Control, vol. 47, no. 10, pp. 1604-1612, Oct. 2002.

[15] X. Mao, J. Lam, S. Xu, and H. Gao, "Razumikhin method and exponential stability of hybrid stochastic delay interval systems," J. Math. Anal. Appl., vol. 314, pp. 45-66, 2006.

[16] M. Mariton, Jump Linear Systems in Automatic Control. New York: Marcel Dekker, 1990.

[17] P. Pepe and Z.-P. Jiang, "A Lyapunov-Krasovskii methodology for ISS and iISS of time-delay systems," Syst. Control Lett., vol. 55, pp. 1006-1014, 2006

[18] A. V. Skorohod, Asymptotic Methods in the Theory of Stochastic Differential Equations. Providence, RI: American Mathematical Society, 1989

[19] E. D. Sontag, "Smooth stabilization implies coprime factorization," IEEE Trans. Automat. Control, vol. AC-34, no. 4, pp. 435-443, Apr. 1989.

[20] E. D. Sontag and Y. Wang, "New characterizations of input-to-state stability," IEEE Trans. Automat. Control, vol. AC-41, no. 9, pp. 1283-1294, Sep. 1996.

[21] D. D. Sworder and R. O. Rogers, "An LQ-solution to a control problem associated with a solar thermal central receiver," IEEE Trans. Automat. Control, vol. AC-28, no. 10, pp. 971-978, Oct. 1983.

[22] A. R. Teel, "Connection between Razumikhin-type theorems and the ISS nonlinear small gain theorem," IEEE Trans. Automat. Control, vol. 43, no. 7, pp. 960-964, Jul. 1998.

[23] B. Xu, "Stability of retarded dynamic systems: A Lyapunov function approach," J. Math. Anal. Appl., vol. 253, pp. 590-615, 2001.

[24] C. Yuan and X. Mao, "Robust stability and controllability of stochastic differential delay equations with Markovian switching," Automatica, vol. 40, pp. 343-354, 2004

[25] D. Yue and Q.-L. Han, "Delay-dependent exponential stability of stochastic systems with time-varying delay, nonlinearity, and Markovian switching," IEEE Trans. Automat. Control, vol. 50, no. 2, pp. 217-222, Feb. 2005.

\section{Signal-to-Noise Ratio Fundamental Limitations in Continuous-Time Linear Output Feedback Control}

\author{
Alejandro J. Rojas, Member, IEEE
}

Abstract-In the present technical note we study the fundamental limitation on stability that arise when an additive coloured Gaussian noise (ACGN) channel is explicitly considered over either the control or measurement paths of a linear time invariant (LTI) feedback loop. By considering a linear setting we can naturally express the fundamental limitation as a lower bound on the channel signal-to-noise ratio (SNR) required for stabilisability. We start by first obtaining a closed-form expression for the squared $L_{2}$ norm of a partial fraction expansion with repeated poles in the Laplace domain. We then use the squared $L_{2}$ norm result to obtain the closed-form expression for the infimal SNR required for stabilisability. The proposed closed-form includes the case of repeated unstable plant poles and non minimum phase (NMP) zeros.

Index Terms-Additive coloured Gaussian noise (ACGN), signal-to-noise ratio (SNR).

\section{INTRODUCTION}

Fundamental limitations in control design as been an important area of research with early seminal results such as [1] and [2]. For a linear time invariant (LTI) plant it is well understood how unstable poles, non minimum phase (NMP) zeros and time-delay cause unavoidable limitations both in regulation and performance (see for example [3] and references therein). In recent years, the study of fundamental limitations has been extended to problems of control over communication networks, [4, Theorem 4.6], [5], attracting a growing interest (see for example [6] and the recent survey by [7]).

Most results in control over network use information theoretic arguments to obtain necessary and sufficient lower bounds on, for example, the transmission data rate for noiseless channels [8], [9] or noisy channels [10]-[13] required for stabilisability. In particular, for linear plant models, in [12, Proposition III.1], it has been shown that if the unstable plant is to be stabilised, then the capacity of a noisy channel in the control loop has to satisfy a lower bound that depends on the unstable eigenvalues of the plant. A similar result was proved earlier in $[8$, Theorem 2.1], for the transmission data rate of a noiseless channel and further extended to nonlinear plant models in [9, Theorem 1].

Another line of research, that does not center on channel capacity nor transmission rate, introduces a framework to study stabilisability of a feedback loop over channels that have a signal to noise ratio (SNR) constraint [14], [15] (and related work in [16], [17]). A recognisable characteristic of the proposed SNR approach is that it is a linear formulation allowing the use of all the linear design techniques (such as cheap control for example). The authors of [14], [18] obtained the expression for the infimal SNR required to stabilise a finite dimensional unstable LTI plant over a memoryless additive white Gaussian noise (AWGN) channel when considering unstable plant poles, NMP zeros and plant time-delay. In [15] the infimal SNR is computed for the case of additive coloured Gaussian noise (ACGN) channels with bandwidth limitation, see Fig. 1. The bandwidth limitation may be imposed for several reasons, for example to avoid interference between different channels in a communication system or to model communications hardware proper-

Manuscript received May 14, 2008; revised December 08, 2008. First published July 24, 2009; current version published August 05, 2009. Recommended by Associate Editor M. Fujita.

The author is with the ARC Centre of Excellence for Complex Dynamic Systems and Control, The University of Newcastle, Newcastle 2308, Australia (e-mail: alejandro.rojas@newcastle.edu.au).

Digital Object Identifier 10.1109/TAC.2009.2022114 


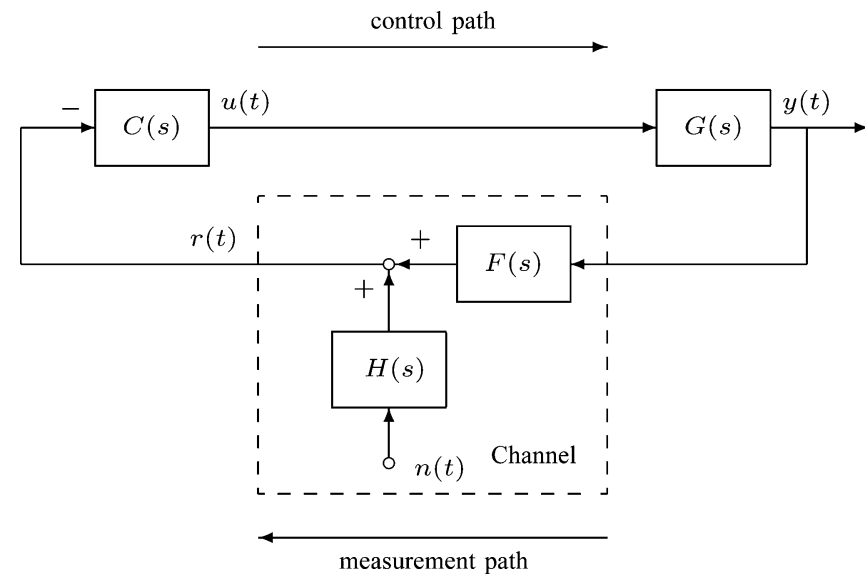

Fig. 1. Linear time invariant (LTI) control system with feedback over an additive coloured Gaussian noise (ACGN) channel with memory.

ties. Furthermore, coloured noise is a more flexible and realistic feature for a communication channel.

The raison d'etre of the present technical note is then to use the SNR approach to study the novel fundamental limitations imposed by the presence of a channel model on the stability of a control loop.

Our first contribution is a technical result that describes in closedform the squared $L_{2}$ norm of a partial fraction expansion in the Laplace domain with repeated poles in the open right-half of the plane.

Our second and main contribution consists of a closed-form expression describing the continuous-time infimal LTI SNR for stabilisability for a, possibly non-minimum phase, ACGN channel with bandwidth limitation and an unstable, possibly non-minimum phase, plant with time-delay. The present infimal SNR result is novel in that considers unstable poles and NMP zeros with multiplicities greater than one, as well as time-delay, revealing in its closed-form the interplay that take place between all the factors causing the observed fundamental limitation.

In the present technical note, as in [14], we neglect all pre- and postsignal processing involved in the communication link, which is then reduced to the communication channel itself.

The present technical note is organised as follows: In Section II we briefly present the squared $L_{2}$ norm closed-form expressions for a partial fraction expansion of unstable poles in the Laplace domain and other technical results. In Section III we apply the squared $L_{2}$ norm for unstable poles results to obtain the closed-form LTI continuous-time infimal SNR constrained solution. Finally, in Section IV we present our final remarks and discuss future research opportunities.

1) Terminology: Let $\mathbb{C}$ denote the complex plane. Let $\mathbb{C}^{-}, \overline{\mathbb{C}}^{-}$, $\mathbb{C}^{+}$and $\overline{\mathbb{C}}^{+}$denote respectively the open-left, closed-left, open-right and closed-right halves of the complex plane $\mathbb{C}$. Let $\mathbb{R}$ denote the set of real numbers, $\mathbb{R}^{+}$the set of positive real numbers, $\mathbb{R}_{o}^{+}$the set of non-negative real numbers and $\mathbb{R}^{-}$the set of real negative numbers. Let $\mathbb{Z}^{+}$denote the set of positive integers. Define $\mathbf{j}=\sqrt{-1}$. A continuoustime signal is denoted by $x(t), t \in \mathbb{R}_{o}^{+}$, and its Laplace transform by $X(s), s \in \mathbb{C}$. The expectation operator is denoted by $\mathcal{E}$. A rational transfer function $P(s)$ of a continuous-time system is termed minimum phase (MP) if all its zeros lie in $\overline{\mathbb{C}}^{-}$, and is non minimum phase (NMP) if it has zeros in $\mathbb{C}^{+}$. The $H_{\infty}$ space is a (closed) subspace of $L_{\infty}$ with functions that are analytic and bounded in $\mathbb{C}^{+}$. The $R H_{\infty}$ space consists of all proper and real rational stable transfer functions. The norm of a system $P(s)$ in $H_{\infty}$ is given by $\|P\|_{\infty}=\sup _{\omega \in \mathbb{R}}|P(\mathbf{j} \omega)|$. Define $L_{2}$ as the space of functions $f: \mathbf{j} \mathbb{R} \rightarrow \mathbb{C}$ such that $\|f\|_{2}^{2}=$ $1 / 2 \pi \int_{-\infty}^{\infty}|f(\mathbf{j} \omega)|^{2} d \omega<\infty$. Define $H_{2}$ as the space of functions $f$ : $\mathbb{C}^{+} \rightarrow \mathbb{C}$ such that $\|f\|_{2}^{2}=\sup _{\sigma>0} 1 / 2 \pi \int_{-\infty}^{\infty}|f(\sigma+\mathbf{j} \omega)|^{2} d \omega<\infty$.
The $H_{2}$ space is a (closed) subspace of $L_{2}$ with functions $f(s)$ analytic in $\mathbb{C}^{+}$. Finally define also $H_{2}^{\perp}$ as the space of functions $f: \mathbb{C}^{-} \rightarrow \mathbb{C}$ such that $\|f\|_{2}^{2}=\sup _{\sigma<0} 1 / 2 \pi \int_{-\infty}^{\infty}|f(\sigma+\mathbf{j} \omega)|^{2} d \omega<\infty$. The $H_{2}^{\perp}$ space is the orthogonal complement of $\mathrm{H}_{2}$ in $L_{2}$, that is the (closed) subspace of functions in $L_{2}$ that are analytic in $\mathbb{C}^{-}$.

\section{Partial Fraction Expansion Technical Results}

We consider in the present section a series of technical results developed in the context of the Laplace domain that are required to obtain the main result in Section III.

Proposition 1: Consider $p \in \mathbb{C}^{-}, n \in \mathbb{Z}^{+}$and $F_{1}(s)$ a transfer function such that $F_{1}(p) \neq \infty$. Then a partial fraction expansion of the expression $F_{1}(s) /\left(s-p_{i}\right)^{n}$ is given by

$$
\frac{F_{1}(s)}{\left(s-p_{i}\right)^{n}}=\frac{c_{n, 1}}{\left(s-p_{i}\right)}+\cdots+\frac{c_{n, n}}{\left(s-p_{i}\right)^{n}}
$$

where

$$
c_{n, k}=\left.\frac{1}{(n-k) !} \frac{d^{n-k}}{d s^{n-k}}\left(F_{1}(s)\right)\right|_{s=p_{i}}, \quad \forall k=1, \ldots, n
$$

are the residues of $F_{1}(s)$ at $s=p_{i}$ with multiplicity $k$.

We follow with a proposition that explicitly states the derivative in the Laplace domain variable $s$ of a pair of differentiable functions as the sum of the derivatives of each such functions.

Proposition 2 (Leibniz's Rule): Consider two differentiable functions $F_{1}(s)$ and $F_{2}(s)$, then the following applies

$$
\begin{aligned}
&\left.\frac{d^{\alpha}}{d s^{\alpha}}\left[F_{1}(s) F_{2}(s)\right]\right|_{s=p_{i}}= \\
&\left.\left.\sum_{k_{o}=0}^{\alpha}\left(\begin{array}{c}
\alpha \\
k_{o}
\end{array}\right) \frac{d^{\alpha-k_{o}} F_{1}(s)}{d s^{\alpha-k_{o}}}\right|_{s=p_{i}} \frac{d^{k_{o}} F_{2}(s)}{d s^{k_{o}}}\right|_{s=p_{i}}
\end{aligned}
$$

with $\alpha \in \mathbb{Z}^{+}$.

The next proposition obtains in closed-form the squared $L_{2}$ norm of a partial fraction expansion with repeated unstable poles.

Proposition 3: Assume $p_{i} \in \mathbb{C}^{+}$to be unstable poles each with multiplicity $n_{i} \in \mathbb{Z}^{+}$, for $i=1, \ldots, m$. Assume also that each related residue $r_{i, l} \in \mathbb{C}$ and that they are known, for $i=1, \ldots, m$ and $l=$ $1, \ldots, n_{i}$. Then

$$
\begin{aligned}
\left\|\sum_{i=1}^{m} \sum_{l=1}^{n_{i}} \frac{r_{i, l}}{\left(s-p_{i}\right)^{l}}\right\|_{2}^{2}= & \sum_{i=1}^{m} \sum_{l=1}^{n_{i}} \frac{r_{i, l}}{(l-1) !} \\
& \left.\sum_{j=1}^{m} \sum_{z=1}^{n_{j}} \frac{d^{l-1}}{d s^{l-1}}\left(\frac{(-1)^{z-1} \bar{r}_{j, z}}{\left(s+\bar{p}_{j}\right)^{z}}\right)\right|_{s=p_{i}}
\end{aligned}
$$

is the closed-form expression for the squared $L_{2}$ norm of the partial fraction expansion $\sum_{i=1}^{m} \sum_{l=1}^{n_{i}} r_{i, l} /\left(s-p_{i}\right)^{l}$.

Proof: Consider the definition for the squared $L_{2}$ norm on the LHS of (4)

$$
\begin{aligned}
\left\|\sum_{i=1}^{m} \sum_{l=1}^{n_{i}} \frac{r_{i, l}}{\left(s-p_{i}\right)^{l}}\right\|_{2}^{2} \\
=\frac{1}{2 \pi} \int_{-\infty}^{\infty}\left(\sum_{i=1}^{m} \sum_{l=1}^{n_{i}} \frac{\bar{r}_{i, l}}{\left(-\mathbf{j} \omega-\bar{p}_{i}\right)^{l}}\right) \\
\quad \times\left(\sum_{i=1}^{m} \sum_{l=1}^{n_{i}} \frac{r_{i, l}}{\left(\mathbf{j} \omega-p_{i}\right)^{l}}\right) d \omega \\
=\frac{1}{2 \pi \mathbf{j}} \int_{\infty \mathbf{j}}^{-\infty \mathbf{j}}\left(\sum_{i=1}^{m} \sum_{l=1}^{n_{i}} \frac{(-1)^{l-1} \bar{r}_{i, l}}{\left(s+\bar{p}_{i}\right)^{l}}\right)
\end{aligned}
$$




$$
\begin{aligned}
& \times\left(\sum_{i=1}^{m} \sum_{l=1}^{n_{i}} \frac{r_{i, l}}{\left(s-p_{i}\right)^{l}}\right) d s \\
= & \frac{1}{2 \pi \mathbf{j}} \oint_{C}\left(\sum_{i=1}^{m} \sum_{l=1}^{n_{i}} \frac{r_{i, l}}{\left(s-p_{i}\right)^{l}}\right) \\
& \times\left(\sum_{i=1}^{m} \sum_{l=1}^{n_{i}} \frac{(-1)^{l-1} \bar{r}_{i, l}}{\left(s+\bar{p}_{i}\right)^{l}}\right) d s .
\end{aligned}
$$

where the inversion of the integrand in the second to last line is such that the right half of the complex plane is the interior of the contour C. In order to apply the Cauchy integral theorem we call on Proposition 1 to identify $c_{l, 1}$ the residue at $s=p_{i}$ for each integrand. Thus the last line above becomes

$$
\begin{aligned}
\left\|\sum_{i=1}^{m} \sum_{l=1}^{n_{i}} \frac{r_{i, l}}{\left(s-p_{i}\right)^{l}}\right\|_{2}^{2}= & \sum_{i=1}^{m} \sum_{l=1}^{n_{i}} \frac{r_{i, l}}{(l-1) !} \\
& \left.\sum_{j=1}^{m} \sum_{z=1}^{n_{j}} \frac{d^{l-1}}{d s^{l-1}}\left(\frac{(-1)^{z-1} \bar{r}_{j, z}}{\left(s+\bar{p}_{j}\right)^{z}}\right)\right|_{s=p_{i}}
\end{aligned}
$$

which ends the proof.

\section{INFIMAL LTI SNR OUTPUT FEEDBACK}

In the present section we apply the $L_{2}$ results for unstable poles in order to obtain the closed-form LTI continuous-time infimal SNR constrained solution. We start by listing the assumptions behind the definition of the infimal SNR for LTI stabilisability problem.

\section{Assumptions}

We list here the general assumptions for the single-input singleoutput (SISO) LTI filters in Fig. 1:

none Plant Model: We assume a continuous-time plant with timedelay defined as

$$
G(s)=G_{1}(s) e^{-s \tau}
$$

where $G_{1}(s)$ is a rational transfer function with relative degree $n_{g} \geq 1$, which contains $m$ unstable poles $\left(p_{i} \in \mathbb{C}^{+}, i=1, \ldots, m\right.$ each with multiplicity $\left.n_{i}\right), q$ NMP zeros $\left(z_{j} \in \mathbb{C}^{+}, j=1, \ldots, q\right.$, each with multiplicity $l_{j}$ and no NMP zeros match any of the $m$ unstable poles) and $\tau \in \mathbb{R}_{o}^{+}$.

none Channel Model: The channel transfer function $F(s)$ modelling the bandwidth limitation and channel dynamics is assumed to be stable, with $f$ NMP zeros $\left(w_{j} \in \mathbb{C}^{+}, j=1, \ldots, f\right.$ each with multiplicity $k_{j}$ and no NMP zeros match any of the $m$ unstable poles). The relative degree of $F(s)$ is denoted by $n_{f} \geq 0$ and the frequency response of $F(s)$ has magnitude 1 inside its bandwidth.

none Channel additive noise process: The channel additive noise process is labelled $n(t)$ and it is a zero-mean Gaussian white noise process with constant power spectral density $\Phi$.

none Noise model: The channel transfer function $H(s)$, colouring the additive white Gaussian noise, is assumed to be stable, minimum phase and with relative degree $n_{h} \geq 0$.

The bandwidth-limited ACGN channel is characterised by two stable transfer functions, $F(s)$ and $H(s)$, and two parameters: The noise spectral density $\Phi$ and the admissible input power level $P$.

Of the two possible locations for the ACGN channel (measurement path and control path), we consider the measurement path location, as in Fig. 1. Nonetheless, in a SISO LTI setting both forms are equivalent, and it is a simple matter to restate the results for the case of actuation performed over a communication channel.

\section{A. Problem Definition}

We restrict our attention to the case where the overall feedback system is stabilised by the LTI controller $C(s)$, see Fig. 1. Denote the power spectral density of $y(t)$ by $S_{y}(\omega)$. Under reasonable stationarity assumptions [19, Section 4.4], the channel input power $\|y\|_{\text {Pow }}^{2}$ may be computed by means of its input spectral density as follows

$$
\|y\|_{\text {Pow }}^{2}=\frac{1}{2 \pi} \int_{-\infty}^{\infty} S_{y}(\omega) d \omega .
$$

The closed-loop transfer function $T_{y n}(s)$, from channel noise $n(t)$ to channel input $y(t)$, is equal to

$$
T_{y n}(s)=-\frac{C(s) G(s)}{1+C(s) G(s) F(s)} H(s) .
$$

If the feedback system is stable, then the power of the channel input signal is given by $\|y\|_{\text {Pow }}^{2}=\left\|T_{y n}\right\|_{2}^{2} \Phi$. We see that the input power constraint may be restated as a constraint imposed on the transfer function (9) by the admissible channel SNR, specifically $P / \Phi>\left\|T_{y n}\right\|_{2}^{2}$. Notice that the proposed SNR involves $\Phi$, the power spectral density of the channel noise and not its power, which is ill-defined in continuous-time (this can be seen by directly replacing $y$ with $n$ in (8)).

Let $\mathcal{K}$ denote the class of all proper controllers $C(s)$ that internally stabilise the feedback system of Fig. 1.

1) Problem 1 (Continuous-Time Infimal SNR for LTI Stabilisability Problem): Find a proper rational function $C(s) \in \mathcal{K}$ such that the transfer function (14) satisfies the constraint imposed by the admissible channel SNR.

\section{B. Main Result}

Denote the Blaschke product containing the unstable poles of $G(s)$ (that is the poles in $\mathbb{C}^{+}$) by

$$
B_{p}(s)=\prod_{i=1}^{m}\left(\frac{s-p_{i}}{s+\bar{p}_{i}}\right)^{n_{i}} .
$$

Denote also the Blaschke product containing the $\overline{\mathbb{C}}^{+}$zeros of $G(s)$ and $F(s)$ as

$$
\begin{aligned}
& B_{z G}(s)=\prod_{j=1}^{q}\left(\frac{s-z_{j}}{s+\bar{z}_{j}}\right)^{l_{j}} \\
& B_{z F}(s)=\prod_{j=1}^{f}\left(\frac{s-w_{j}}{s+\bar{w}_{j}}\right)^{k_{j}} .
\end{aligned}
$$

In general, if it is not necessary to stress the different sources of possible NMP zeros we will use the short-hand notation $B_{z}(s)$, where $B_{z}(s)=$ $B_{z G}(s) B_{z F}(s)$ and define $\tilde{F}(s)=B_{z F}^{-1}(s) F(s)$, the channel model transfer function with all the NMP zeros replaced by their minimum phase counterparts.

Theorem 4 (Continuous-Time Infimal SNR for Stabilisability): Consider the output LTI feedback represented in Fig. 1 and $G(s), F(s)$ and $H(s)$ as in Section III-A, then

$$
\frac{P}{\Phi}>\left.\sum_{i=1}^{m} \sum_{l=1}^{n_{i}} \frac{r_{i, l}}{(l-1) !} \sum_{j=1}^{m} \sum_{z=1}^{n_{j}} \frac{d^{l-1}}{d s^{l-1}}\left(\frac{(-1)^{z-1} \bar{r}_{j, z} e^{\left(p_{i}+\bar{p}_{j}\right) \tau}}{\left(s+\bar{p}_{j}\right)^{z}}\right)\right|_{s=p_{i}}
$$

in which we have

$$
\begin{aligned}
& r_{i, l}=\frac{1}{\left(n_{i}-l\right) !} \sum_{k=l}^{n_{i}}\left(\begin{array}{c}
n_{i}-l \\
k-l
\end{array}\right) \tau^{k-l} \\
& \left.\cdot \frac{d^{n_{i}-k}}{d s^{n_{i}-k}}\left(\left(s-p_{i}\right)^{n_{i}} B_{p}^{-1}(s) B_{z}^{-1}(s) \tilde{F}^{-1}(s) H(s)\right)\right|_{s=p_{i}}
\end{aligned}
$$


Proof: Consider a coprime factorisation for $F(s) G(s)$ as

$$
F(s) G(s)=\frac{e^{-s \tau} N(s)}{M(s)}
$$

where $N(s), M(s) \in R H_{\infty}$. Further, without loss of generality, consider

$$
N(s)=B_{z}(s) N_{o}(s) \tilde{F}(s), \quad M(s)=B_{p}(s) M_{o}(s)
$$

where $N_{o}(s), M_{o}(s) \in R H_{\infty}, N_{o}(s)$ and $M_{o}(s)$ are stable and minimum phase transfer functions, $B_{p}(s), B_{z}(s)$ are the Blaschke products containing the plant unstable poles and NMP zeros respectively and $\tilde{F}(s)=B_{z F}^{-1}(s) F(s)$. Furthermore, with no loss of generality, consider $N_{o}(s)$ with relative degree $n_{g}$ (the same as $G_{1}(s)$ in (7)) and $M_{o}(s)$ with relative degree zero.

Applying a technique used in [20] and following [18, Lemma 3.1], a Youla parameterisation of all controllers that stabilise $G(s)$ is given by

$$
C(s)=\frac{X(s)+M(s) Q(s)}{Y(s)-e^{-s \tau} N(s) Q(s)}
$$

where $X(s)$ is in $R H_{\infty}, Q(s), Y(s)$ are in $H_{\infty}$ and $X(s)$ and $Y(s)$ satisfy the Bezout identity

$$
e^{-s \tau} N(s) X(s)+M(s) Y(s)=1 .
$$

A demonstration of the above Bezout identity (17) can be found for example in [21, Lemma 3.2]. Replacing these factorisations for $F(s) G(s)$ and $C(s)$ into $T_{y n}$ as in (9) gives

$$
\begin{aligned}
T_{y n}(s)=-\left(e^{-s \tau} B_{z}(s) N_{o}(s) \tilde{F}(s) X(s)+e^{-s \tau} B_{p}(s)\right. \\
\left.\cdot B_{z}(s) M_{o}(s) N_{o}(s) \tilde{F}(s) Q(s)\right) F^{-1}(s) H(s) .
\end{aligned}
$$

Since $B_{p}(s)$ and $B_{z}(s)$ (as well as $B_{z G}(s)$ and $B_{z F}(s)$ ) are all pass, they have everywhere magnitude one, thus we can extract them from the definition of the squared $H_{2}$ norm of $T_{y n}(s)$ and obtain

$$
\begin{aligned}
\inf _{Q(s) \in H_{\infty}} & \left\|T_{y n}\right\|_{2}^{2} \\
= & \inf _{Q(s) \in H_{\infty}}\left\|e^{-s \tau} B_{p}^{-1} N_{o} X H+e^{-s \tau} M_{o} N_{o} Q H\right\|_{2}^{2} .
\end{aligned}
$$

Since $e^{-s \tau}$ has magnitude one at all frequencies, the norm expression on the RHS of (19) is not affected by it

$$
\inf _{Q(s) \in H_{\infty}}\left\|T_{y n}\right\|_{2}^{2}=\inf _{Q(s) \in H_{\infty}}\left\|B_{p}^{-1} N_{o} X H+M_{o} N_{o} Q H\right\|_{2}^{2} .
$$

The first term inside the RHS norm expression in (20) is a mixed term with stable and unstable poles. Notice that this term is also square integrable since $N_{o}(s)$ has relative degree $n_{g} \geq 1, X(s)$ is proper since it is in $R H_{\infty}$ and $B_{p}^{-1}(s), H(s)$ have both relative degree zero. In a similar way, the second term inside the RHS norm expression in (20) is clearly a stable term and also square integrable since $M_{o}(s)$ and $H(s)$ have relative degree zero, $N_{o}(s)$ has relative degree $n_{g} \geq 1$ and $Q(s)$ is proper since it is an element of $H_{\infty}$. To continue the proof consider the fact that the term $B_{p}^{-1}(s) N_{o}(s) X(s) H(s)$ can be decomposed as

$$
B_{p}^{-1}(s) N_{o}(s) X(s) H(s)=\Gamma^{\perp}(s)+\Gamma(s)
$$

where, by definition, $\Gamma(s)$ is in $H_{2}$, whilst $\Gamma^{\perp}(s)$ is in $H_{2}^{\perp}$ and therefore by Lemma 3 in [22, Section 10.4],

$$
\inf _{Q(s) \in H_{\infty}}\left\|T_{y n}\right\|_{2}^{2}=\left\|\Gamma^{\perp}\right\|_{2}^{2}+\inf _{Q(s) \in H_{\infty}}\left\|\Gamma+M_{o} N_{o} Q H\right\|_{2}^{2} .
$$

By means of a partial fraction expansion and the Bezout identity in (17), it is possible to quantify $\Gamma^{\perp}(s)$ as

$$
\Gamma^{\perp}(s)=\sum_{i=1}^{m} \sum_{l=1}^{n_{i}} \frac{\tilde{r}_{i, l}}{\left(s-p_{i}\right)^{l}}
$$

where by applying Proposition 1 we obtain

$$
\begin{aligned}
& \tilde{r}_{i, l}=\frac{1}{\left(n_{i}-l\right) !} . \\
& \left.\cdot \frac{d^{n_{i}-l}}{d s^{n_{i}-l}}\left(\left(s-p_{i}\right)^{n_{i}} B_{p}^{-1}(s) N_{o}(s) X(s) H(s)\right)\right|_{s=p_{i}} .
\end{aligned}
$$

To avoid the dependency on $X(s)$ in $\tilde{r}_{i, l}$ we use Proposition 2 where $\left(s-p_{i}\right)^{n_{i}} B_{p}^{-1}(s) B_{z}^{-1}(s) \tilde{F}^{-1}(s) H(s)$ plays the role of $F_{1}(s), N(s) X(s)$ of $F_{2}(s), \alpha=n_{i}-l$ and $k_{o}=k-l$. Furthermore, from the Bezout identity defined in (17) we also notice that $d^{k-l}[N(s) X(s)] /\left.d s^{k-l}\right|_{s=p_{i}}=\tau^{k-l} e^{p_{i} \tau}$. Finally we can then recognise $\tilde{r}_{i, l}=r_{i, l} e^{p_{i} \tau}$, where the term $r_{i, l}$ is given by

$$
\begin{aligned}
& r_{i, l}=\frac{1}{\left(n_{i}-l\right) !} \sum_{k=l}^{n_{i}}\left(\begin{array}{c}
n_{i}-l \\
k-l
\end{array}\right) \tau^{k-l} . \\
& \left.\cdot \frac{d^{n_{i}-k}}{d s^{n_{i}-k}}\left(\left(s-p_{i}\right)^{n_{i}} B_{p}^{-1}(s) B_{z}^{-1}(s) \tilde{F}^{-1}(s) H(s)\right)\right|_{s=p_{i}} .
\end{aligned}
$$

The result for the first squared $H_{2}^{\perp}$ norm term on the RHS of (22), by use of the Residue theorem (see for example [23, pp. 169-172]) as proposed in Proposition 3, is then

$$
\begin{aligned}
& \left\|\Gamma^{\perp}\right\|_{2}^{2}= \\
& \left.\sum_{i=1}^{m} \sum_{l=1}^{n_{i}} \frac{r_{i, l}}{(l-1) !} \sum_{j=1}^{m} \sum_{z=1}^{n_{j}} \frac{d^{l-1}}{d s^{l-1}}\left(\frac{(-1)^{z-1} \bar{r}_{j, z} e^{\left(p_{i}+\bar{p}_{j}\right) \tau}}{\left(s+\bar{p}_{j}\right)^{z}}\right)\right|_{s=p_{i}} .
\end{aligned}
$$

Replacing in (22) gives

$$
\begin{aligned}
& \inf _{Q(s) \in H_{\infty}}\left\|T_{y n}\right\|_{2}^{2}= \\
& \left.\sum_{i=1}^{m} \sum_{l=1}^{n_{i}} \frac{r_{i, l}}{(l-1) !} \sum_{j=1}^{m} \sum_{z=1}^{n_{j}} \frac{d^{l-1}}{d s^{l-1}}\left(\frac{(-1)^{z-1} \bar{r}_{j, z} e^{\left(p_{i}+\bar{p}_{j}\right) \tau}}{\left(s+\bar{p}_{j}\right)^{z}}\right)\right|_{s=p_{i}}+ \\
& \inf _{Q(s) \in H_{\infty}}\left\|\Gamma+M_{o} N_{o} Q H\right\|_{2}^{2} .
\end{aligned}
$$

We can make the expression $\Gamma(s)+M_{o}(s) N_{o}(s) Q(s) H(s)$ arbitrarily small in norm by choosing $Q(s)$ as

$$
\hat{Q}(s)=-M_{o}^{-1}(s) N_{o}^{-1}(s) \Gamma(s) H^{-1}(s) .
$$

If the appropriate $\hat{Q}(s)$ is not proper then consider the choice $Q_{\varepsilon}(s)=$ $\hat{Q}(s) /(\varepsilon s+1)^{\gamma}$, with $\gamma \in \mathbb{Z}^{+}$and apply Lemma 10 from [24, p.171]. As $\varepsilon \rightarrow 0$ we obtain the infimal squared norm that can be achieved in (25) as

$$
\begin{aligned}
& \inf _{Q(s) \in H_{\infty}}\left\|T_{y n}\right\|_{2}^{2}= \\
& \left.\sum_{i=1}^{m} \sum_{l=1}^{n_{i}} \frac{r_{i, l}}{(l-1) !} \sum_{j=1}^{m} \sum_{z=1}^{n_{j}} \frac{d^{l-1}}{d s^{l-1}}\left(\frac{(-1)^{z-1} \bar{r}_{j, z} e^{\left(p_{i}+\bar{p}_{j}\right) \tau}}{\left(s+\bar{p}_{j}\right)^{z}}\right)\right|_{s=p_{i}}
\end{aligned}
$$

which completes the proof.

Remark 1: Notice from Theorem 4 that we regain the result of Theorem 1 in [15] whenever $n_{i}=1, i=1, \ldots, m$, that is

$$
\frac{P}{\Phi}>\sum_{i=1}^{m} \sum_{j=1}^{m} \frac{r_{i} \bar{r}_{j}}{p_{i}+\bar{p}_{j}} e^{\left(p_{i}+\bar{p}_{j}\right) \tau}
$$

where $r_{i}=r_{i, 1}$ and $\bar{r}_{j, 1}=\bar{r}_{j}$, in order to simplify the notation. 


\section{Discussion}

Consider the simple case of a double unstable pole $p$, for a plant and bandwidth limited ACGN channel as in Section III-A. We have then, from Theorem 4, that the infimal SNR for stabilisability is given by

$$
\frac{P}{\Phi}>\left(\frac{r_{1,1}^{2}}{2 p}-\frac{2 r_{1,1} r_{1,2}}{(2 p)^{2}}+\frac{2 r_{1,2}^{2}}{(2 p)^{3}}\right) e^{2 p \tau}
$$

and the two residues involved in the above expression are given by

$$
\begin{aligned}
r_{1,1}= & 2(2 p) B_{z}^{-1}(p) \tilde{F}^{-1}(p) H(p) \\
& +\left.(2 p)^{2} \frac{d}{d s}\left(B_{z}^{-1}(s)\right)\right|_{s=p} \tilde{F}^{-1}(p) H(p) \\
& +\left.(2 p)^{2} B_{z}^{-1}(p) \frac{d}{d s}\left(\tilde{F}^{-1}(s)\right)\right|_{s=p} H(p) \\
& +\left.(2 p)^{2} B_{z}^{-1}(p) \tilde{F}^{-1}(p) \frac{d}{d s}(H(s))\right|_{s=p} \\
& +\tau(2 p)^{2} B_{z}^{-1}(p) \tilde{F}^{-1}(p) H(p) \\
r_{1,2}= & (2 p)^{2} B_{z}^{-1}(p) \tilde{F}^{-1}(p) H(p) .
\end{aligned}
$$

We observe from this that the residue factor $r_{1,1}$ will then grow linearly as a function of the delay $\tau$. Such insight could not have been obtained from [15, Theorem 1], if for example the repeated pole was approximated by a set of two nearly multiple poles. We can also observe that, lacking a transfer function description for $F(s)$ and $H(s)$, any approximating function needs to be at least differentiable once in " $s$ " if we are to compute the infimal SNR for stabilisability expressed in (28).

1) Example 1: In the present example the plant and channel model are given by

$$
\begin{aligned}
& G(s)=\frac{e^{-s \tau}}{(s-p)^{2}} \\
& F(s)=\frac{(s-z)}{(s+z)(a s+1)}, \quad H(s)=1 .
\end{aligned}
$$

Thus we can recognise $B_{p}(s)=(s-p / s+p)^{2}, B_{z}(s)=$ $s-z / s+z$ and $\tilde{F}(s)=1 /(a s+1)$. From Theorem 4 we have that

$$
\begin{aligned}
r_{1,1}= & 2 p \frac{\left(2(2 a p+1)\left(p^{2}-z^{2}\right)-4 p z(a p+1)\right)}{(p-z)^{2}} \\
& +\tau(2 p)^{2}\left(\frac{p+z}{p-z}\right)(a p+1) \\
r_{1,2}= & (2 p)^{2}\left(\frac{p+z}{p-z}\right)(a p+1) .
\end{aligned}
$$

Also, from Theorem 4 we have that the infimal SNR for stabilisability is given by (28). We can observe directly from the residue factors how:

1) As the value of $p \rightarrow 0$ the infimal SNR for stabilisability tends to zero.

2) As the value of $p$ approaches the value of $z$, the infimal SNR for stabilisability will tend to infinity due to the factor $(p-z)$ in the denominator of each residue.

3) As $p \rightarrow \infty$ the infimal SNR for stabilisability also tends to infinity.

4) The presence of the time-delay $\tau$ increases the infimal SNR for stabilisability required for stabilisability through the factor $e^{2 p \tau}$ and linearly in $r_{1,1}$.

5) Since the bandwidth of the channel model $F(s)$ is represented by $a$, we can observe (for $z=0$ ) that the less bandwidth available (that is $a \rightarrow \infty$ ), for any given value of $p, z$ and $\tau$, the greater the infimal SNR for stabilisability will be.

6) The residue expressions $r_{1,1}$ and $r_{1,2}$ are in closed-form and can be explicitly obtained solving the derivatives for $B_{p}(s), B_{z}(s)$ and $\tilde{F}(s)$ from the general definition presented in (13).
From the previous example and the expression for $r_{1,1}$ in (29) we can also observe that if $p$ is well outside the bandwidth of $\tilde{F}(s)$ then $\tilde{F}(p) \approx 0$ and $\tilde{F}^{-1}(p) \approx \infty$, whilst $d \tilde{F}^{-1}(s) /\left.d s\right|_{s=p} \approx 0$ (assuming that the frequency response of $\tilde{F}(s)$ flattens out appropriately in the vicinity of $p$ ). On the other hand, as long as $p$ is well inside the bandwidth of $\tilde{F}(s)$ then $\tilde{F}(p) \approx 1, \tilde{F}^{-1}(p) \approx 1$, and $d \tilde{F}^{-1}(s) /\left.d s\right|_{s=p} \approx$ 0 (assuming again that the frequency response of $\tilde{F}(s)$ flattens out appropriately in the vicinity of $p$ ). Thus, we can summarize the present observation as follows: "If the unstable poles of the plant model are outside the channel bandwidth, the SNR limitation will be severe".

In some cases this observation can also be used to obtain a necessary condition for stabilisability, that might help reduce the calculations involved.

Remark 2: The infimal SNR for stabilisability due to an AWGN channel model with bandwidth limitation is lower bounded by the infimal SNR for stabilisability due to a memoryless AWGN channel.

Corollary 5 (Infimal SNR for Stabilisability Over a Memoryless AWGN Channel): Consider a minimum phase plant with one real unstable pole $p$ of multiplicity $n_{1}$ and time-delay $\tau$. As a result of Theorem 4 and Remark 2, a necessary condition for stabilisability of such a plant is given by

$$
\frac{P}{\Phi}>\sum_{l=1}^{n_{1}} r_{1, l} \sum_{z=1}^{n_{1}}\left(\begin{array}{c}
z+l-2 \\
z-1
\end{array}\right) \frac{(-1)^{z+l-2} \bar{r}_{1, z}}{(2 p)^{z+l-1}} e^{(p+\bar{p}) \tau}
$$

with

$$
r_{1, l}=\sum_{k=l}^{n_{1}}\left(\begin{array}{c}
n_{1} \\
k
\end{array}\right) \frac{\tau^{k-l}}{(k-l) !}(2 p)^{k} .
$$

The importance of Corollary 5 resides in the fact that, in light of Remark 2, it gives an expression that in general is of easier computation for a rapid estimation of the seriousness of the fundamental limitation. We stress though that for AWGN channels with bandwidth limitation, the result of Corollary 5 is necessary but not sufficient for stabilisability. We can also observe from Corollary 5 that the infimal SNR for stabilisability requirement is dominated by a term of the form $(2 p)^{2 n_{1}-1} \tau^{2 n_{1}-2} e^{2 p \tau} /\left(\left(n_{1}-1\right) !\right)^{2}$. As a side note, observing the case for $\tau=0$, from Corollary 5 we obtain

$$
\frac{P}{\Phi}>2 p \sum_{l=1}^{n_{1}}\left(\begin{array}{c}
n_{1} \\
l
\end{array}\right) \sum_{z=1}^{n_{1}}\left(\begin{array}{c}
z+l-2 \\
z-1
\end{array}\right)\left(\begin{array}{c}
n_{1} \\
z
\end{array}\right)(-1)^{z+l-2} .
$$

Since for this case we must recover a lower bound of $2 p n_{1}$ (see for example [14, Theorem II.1]) we notice then that we have also indirectly proved that

$$
\sum_{l=1}^{n_{1}} \sum_{z=1}^{n_{1}}\left(\begin{array}{c}
n_{1} \\
l
\end{array}\right)\left(\begin{array}{c}
z+l-2 \\
z-1
\end{array}\right)\left(\begin{array}{c}
n_{1} \\
z
\end{array}\right)(-1)^{z+l-2}=n_{1}
$$

which can be verified numerically to hold true. A direct theoretical proof of the equality in (34) is outside the scope of the present technical note. Some in road although can be obtained via combinatorial equalities involving the Kronecker delta, see for example [25, p. 84].

We conclude the present subsection with a more general remark on the optimality of the linear controller solution versus nonlinear, or indeed time-varying solutions.

Remark 3: The optimality of the linear approach, in terms of channel capacity, as been shown to hold for the case of discrete-time minimum phase unstable plant models with relative degree one and AWGN channel models, see for example [14], as well as single pole unstable minimum phase plant models with relative degree 1 and an MA1 ACGN channel model, see [26]. 
Nonetheless, as a consequence of restricting the stabilising controller to a linear transfer function, we have to accept that the obtained infimal SNR for stabilisability might be sub-optimal. That is, there might be a nonlinear or time varying stabilising solution that achieves a lower SNR. This fact, more so in a discrete-time context, is tightly linked to achieving the infimal channel capacity for stabilisability, [12], where for a memoryless AWGN channel no linearity is indeed assumed for the encoder and decoder stages stabilising the plant. We also refer to [27] where the Witsenhausen's counterexample has been extended to include a simplified channel model, showing that nonlinear strategies might outperform the best linear strategy in the context of control over networks.

\section{CONCLUSION}

In the present technical note we have developed a closed-form expression for the squared $L_{2}$ norm of a partial fraction expansion that explicitly considers repeated unstable poles. We have then applied the squared $L_{2}$ norm for unstable repeated poles result to the continuous-time infimal LTI SNR for stabilisability problem. We obtained in closed-form the infimal LTI SNR lower bound for stabilisability, which is both necessary and sufficient and when considering single unstable poles and single NMP zeros, it agrees with earlier results such as [15].

The infimal LTI SNR lower bound expression shows all the elements that hinder achieving closed-loop stability. On the plant model side we have the contribution of unstable poles, NMP zeros and time-delay. On the channel model side we have the contribution of NMP zeros, the bandwidth limitation imposed by the LTI filter $F(s)$, and the channel noise colouring by the LTI filter $H(s)$. We have seen that a quasi-cancellation of an NMP zero and an unstable pole is particularly deleterious in terms of the required infimal SNR for stabilisability, in line with the requirement for the closed-loop to be internally stable.This effect is captured by the inverse of the NMP zeros Blaschke product $B_{z}(s)$ in the definition of the residue factors $r_{i, l}$, which when evaluated at the culprit pole location will tend to infinity. Also, we have observed the interplay between the time delay, the unstable poles of the plant and their multiplicities.

Further directions of research will consider the extension of the work presented here to multivariable systems and to performance problems.

\section{REFERENCES}

[1] H. Bode, Network Analysis and Feedback Amplifier Design. Princeton, NJ: Van Nostrand, 1945.

[2] I. Horowitz, Synthesis of Feedback Systems. New York: Academic Press, 1963.

[3] M. Seron, J. Braslavsky, and G. Goodwin, Fundamental Limitations in Filtering and Control. New York: Springer, 1997.

[4] N. Elia, "When bode meets Shannon: Control-oriented feedback communication schemes," IEEE Trans. Automat. Control, vol. 49, no. 9, pp. 1477-1488, Sep. 2004.

[5] N. C. Martins and M. A. Daleh, "Feedback control in the presence of noisy channels: Bode-like fundamental limitations of performance," IEEE Trans. Automat. Control, vol. 53, no. 7, pp. 1604-1615, Aug. 2008.

[6] P. Antsaklis and J. Baillieul, Eds., "Special Issue on Technolgoy Networked Control Systems," Proc. IEEE, vol. 49, no. 9, Sep. 2004.

[7] G. Nair, F. Fagnani, S. Zampieri, and R. Evans, "Feedback control under data rate constraints: An overview," Proc. IEEE (Special Issue on The Emerging Technology of Networked Control Systems), vol. 95, no. 1, pp. 108-137, Jan. 2007.

[8] G. Nair and R. Evans, "Stabilizability of stochastic linear systems with finite feedback data rates," SIAM J. Control Optim., vol. 43, no. 2, pp. 413-436, Jul. 2004.
[9] G. Nair, R. Evans, I. Mareels, and W. Moran, "Topological feedback entropy and nonlinear stabilization," IEEE Trans. Automat. Control, vol. 49, no. 9, pp. 1585-1597, Sep. 2004.

[10] A. Matveev and A. Savkin, "An analogue of shannon information theory for networked control systems. Stabilization via a noisy discrete channel," in Proc. 43th IEEE CDC, Paradise Island, Bahamas, 2004, pp. 4491-4496.

[11] S. Tatikonda and S. Mitter, "Control over noisy channels," IEEE Trans. Automat. Control, vol. 49, no. 7, pp. 1196-1201, Jul. 2004.

[12] J. Freudenberg, R. Middleton, and V. Solo, "The minimal signal-tonoise ratio required to stabilize over a noisy channel," in Proc. Amer. Control Conf., Minneapolis, MN, 2006, pp. 650-655.

[13] C. Charalambous and A. Farhadi, "Control of feedback systems subject to the finite rate constraints via the Shannon lower bound," in Proc. 5th Int. Symp. Modeling Optim. Mobile, Ad Hoc, Wireless Networks, Limassol, Cyprus, 2007, pp. 1-7.

[14] J. Braslavsky, R. Middleton, and J. Freudenberg, "Feedback stabilisation over signal-to-noise ratio constrained channels," IEEE Trans. Automat. Control, vol. 52, no. 8, pp. 1391-1403, Aug. 2007.

[15] A. Rojas, J. Braslavsky, and R. Middleton, "Output feedback stabilisation over bandwidth limited, signal to noise ratio constrained communication channels," in Proc. Amer. Control Conf., Minneapolis, MN, Jun. 2006, pp. 2789-2794.

[16] B. Bassam and P. Voulgaris, "A convex characterization of distributed control problems in spatially invariant systems with communication constraints," Syst. Control Lett., vol. 54, pp. 574-583, 2005.

[17] A. Rantzer, "Linear quadratic team theory revisited," in Proc. Amer. Control Conf., Minneapolis, MN, 2006, pp. 1637-1641.

[18] J. Braslavsky, R. Middleton, and J. Freudenberg, "Effects of time delay on feedback stabilization over signal-to-noise ratio constrained channels," in Proc. 16th IFAC World Congress, Jul. 2005, pp. 219-224.

[19] K. Åström, Introduction to Stochastic Control Theory. New York: Academic Press, 1970.

[20] J. Chen, L. Qiu, and O. Toker, "Limitations on maximal tracking accuracy," IEEE Trans. Automat. Control, vol. 45, no. 2, pp. 326-331, Feb. 2000.

[21] G. Meinsma and H. Zwart, "On $H_{\infty}$ control for dead-time systems," IEEE Trans. Automat. Control, vol. 45, no. 2, pp. 272-285, Feb. 2000.

[22] J. Doyle, B. Francis, and A. Tannenbaum, Feedback Control Theory. London, U.K.: Macmillan Publishing Company, 1992.

[23] R. Churchill and J. Brown, Complex Variables and Applications, 5th ed. New York: McGraw-Hill, 1990.

[24] M. Vidyasagar, Control System Synthesis: A Factorization Approach. Cambridge, MA: MIT Press, 1985.

[25] A. Benjamin and J. Quinn, Proofs That Really Count: The Art of Combinatorial Proof, ser. Dolciani Mathematical Expositions. Washington, DC: The Mathematical Association of America, 2003.

[26] R. Middleton, A. Rojas, J. Freudenberg, and J. Braslavsky, "Feedback stabilization over a first order moving average Gaussian noise channel," IEEE Trans. Automat. Control, vol. 54, no. 1, pp. 163-167, Jan. 2009.

[27] N. Martins, "Witsenhausen's counter example holds in the presence of side information," in Proc. 45th IEEE Conf. Decision Control, San Diego, CA, 2006, pp. 1111-1116. 